\title{
ELEMENTOS DE FILIACION CAMPANIFORME EN LAS ISLAS BALEARES: VALORACION Y SIGNIFICADO CULTURAL
}

\author{
MANUEL FERNÁNDEZ-MIRANDA
}

El descubrimiento de elementos campaniformes en las Islas Baleares es relativamente antiguo, pues se remonta al hallazgo de un fragmento cerámico decorado a base de dientes de lobo y líneas continuas que se encontró en la Cova dels Bous de Santueri, Felanitx, a principios de siglo. Castillo, en su conocida síntesis sobre el vaso campaniforme, lo usó para crear el llamado "grupo balear", constituido por esa única pieza y, según él, vinculado al septentrional de Cerdeña (Castillo, 1928).

A partir de los años cincuenta los hallazgos de cerámicas de estilo campaniforme en Mallorca se multiplicaron. Una primera síntesis de las entonces llamadas cerámicas con decoración incisa se debe a Roselló Bordoy (1960), a la que seguiría, poco más de una década después, la monografía exhaustiva de Cantarellas (1972 a), donde se estudian todas esas cerámicas y sus contextos arqueológicos, si bien restándoles importancia a la hora de interpretar el conjunto ergológico, en parte por las escasas noticias que hasta entonces se tenían a propósito de la forma en que habían sido hallados los distintos objetos: en la mayoría de los casos o se trataba de hallazgos fortuitos o procedían de excavaciones realizadas con una metodología harto heterodoxa. Dos nuevos yacimientos que han sido excavados en estos últimos años, Son Matge y Son Ferrandell-Oleza, el primero un abrigo rocoso y el segundo un poblado al aire libre, permiten replantear con nuevas maneras el panorama de los elementos culturales campaniformes en Mallorca, y desde allí valorar adecuadamente la presencia de algunos de ellos en otras islas del archipiélago.

\section{Isla de Mallorca}

Entre datos seguros y otros más discutibles se conocen en la actualidad casi una veintena de yacimientos en esta isla donde hayan aparecido cerámicas con motivos decorativos incisos, que se fechen, grosso modo, en época pretalayótica. Tradicionalmente tales yacimientos han venido siendo tratados de manera unitaria, pero un análisis pormenorizado de los mismos permite discernir que, bajo esa etiqueta común, aparecen sitios de muy distinta categoría y, sin duda, filiación cultural y cronológi- 
ca. Varios de esos yacimientos son muy difíciles de clasificar, y algunos imposible, y sus hallazgos más característicos parecen señalar que se trata de productos de época posterior a la que se corresponde con los tipos campaniformes bien fechados; podría asimismo ocurrir en alguna ocasión que simplemente se tratara de productos de baja calidad, imitaciones u obras descuidadas sincrónicas, o bien que deliberadamente se apartaran de los modelos más repetidos, pero mantuvieran la sincronía. Es el caso, por ejemplo, de sitios como C'an Massac, Sa Sinía, Coval Simó, Son Maiol... o Son Sunyer 7, todos ellos yacimientos funerarios claramente pretalayóticos, dentro del grupo que Veny sistematizara hace ya algunos años (Veny, 1968), con piezas que poseen decoraciones incisas pero que en ningún caso deben ser consideradas de carácter campaniforme desde un punto de vista estrictamente tipológico.

De tal manera que, en el momento actual de la investigación, tan sólo siete yacimientos entre los publicados - pues existen noticias de algunos otros todavía inéditos - deben ser clasificados, totalmente o por parte de su secuencia, como característicamente campaniformes: Cova dels Bous, Son Torrella, Son Gallard, Ca Na Cotxera, Son Matge, Son Ferrandell-Oleza y Es Velar de Santanyí. Los dos primeros ofrecen pocas posibilidades para la investigación, pues se trata de hallazgos prácticamente casuales; los cinco restantes son en un caso una cueva natural de enterramiento, Son Gallard, y en los otros cuatro sitios de habitación: Son Matge es un abrigo natural, $\mathrm{Ca} \mathrm{Na}$ Cotxera una habitación aislada al aire libre y Son Ferrandell-Oleza y Es Velar de Santany, este último conocido sólo por prospecciones superficiales, son poblados.

Son Gallard es un abrigo rocoso en la costa norte de Mallorca, cerca de la villa de Deià. Su excavación (Waldren, 1982) proporcionó una secuencia estratigráfica bastante alterada en cuyo nivel inferior aparecieron enterramientos asociados a cerámicas con decoración de tipo campaniforme. Tales enterramientos se encontraban parcialmente destruidos a causa de la utilización del covacho en épocas posteriores, incluyendo unos muros levantados en tiempos modernos para utilizar el lugar como aprisco. No obstante lo hallado permitió comprobar que se trataba de individuos que fueron enterrados acostados sobre su lado derecho, y quizá en posición encogida, en el interior de cistas hechas con lajas de piedra y con fragmentos de cerámica campaniforme como único ajuar. Restos de carbón vegetal extraídos del mismo nivel que los enterramientos, donde también había cerámicas de estilo campaniforme, dieron una fecha C-14 para esa primera ocupación del lugar de 1840 a. C. (Y-1789: $3790 \pm 80$, en Fernández-Miranda y Waldren, 1979, págs. 349 y ss.). Sólo uno de los dos cráneos fue analizado. Corresponde a un individuo masculino hiperbraquicráneo (índice 85,5) de aproximadamente unos 35 años de edad. La presencia de individuos braquicráneos en yacimientos mallorquines del segundo milenio a. C. está también constatada en la cueva de enterramiento de Son Mulet, si bien la mayoría de la población de eas época resulta ser dolicocránea, con las naturales reservas dado el bajo número de individuos hasta ahora analizados (Fernández-Miranda y Garralda, 1978, págs. 87 y ss.).

Distinto interés presenta el sitio de $\mathrm{Ca} \mathrm{Na}$ Cotxera, en el término de Muro, a unos kilómetros de la costa de la bahía de Alcudia. Se trata, aparentemente, de 
una vivienda aislada con una estructura que tiende a la forma cuadrada en el exterior, mientras que al interior los paramentos de los muros se redondean en las esquinas y levanta una pilastra central para sostén de la cubierta. La excavación (Cantarellas, 1972 b) manifestó dos niveles prehistóricos, uno, el denominado b, de clara filiación pretalayótica y fecha absoluta 1800 a. C. (I-5515: $3750 \pm 120$, en FernándezMiranda y Waldren, 1979, págs. 349 y ss.), y otro inferior, el nivel c, que se corresponde con la ocupación original del sitio y que proporcionó una excelente colección de fragmentos cerámicos con decoración de estilo campaniforme, además de vasos carenados y troncocónicos, varios botones con perforación en $\mathrm{V}$ y un buen número de lascas junto a algunas hojas retocadas de sílex. La fauna estaba compuesta por cápridos, bóvidos y moluscos.

$\mathrm{Ca} \mathrm{Na}$ Cotxera debe ser interpretada como un lugar de habitación probablemente aislado, si bien se desconoce si las zonas inmediatas a la excavada fueron objeto de prospección. A la luz de lo publicado parece una cabaña solitaria que aprovechó para su instalación un pequeño montículo en medio de un territorio aluvial muy favorable para el desarrollo de la agricultura. Su fecha debe colocarse, obviamente, en los primeros años del segundo milenio a. C., con cerámicas de tipo campaniforme que responden a los tipos de cuenco y escudilla, y ausencia de productos metálicos, que sí aparecen, aunque en número escaso, en el nivel pretalayótico superior, al que corresponde la datación absoluta citada más arriba.

El otro yacimiento de habitación conocido en la zona baja de la isla es el de Es Velar de Santanyí, si bien la información disponible es muy deficiente dado que procede de trabajos de aficionados locales, al parecer siempre a partir de rebuscas superficiales. Se trata, según los datos disponibles (Carreras y Covas, 1984), de un poblado situado en una zona apta para la ganadería y el cultivo cerealístico en el que se perciben restos de ocupación de diferentes épocas. Una zona del poblado está ocupada casi exclusivamente por restos del estilo de referencia. Los fragmentos cerámicos de tipo campaniforme más significativos presentan motivos de ajedrezado, líneas en zig-zag o dientes de lobo y puntuaciones alternas; parecen corresponder siempre a formas sencillas tipo cuenco. Junto a ellas se recogieron cerámicas lisas carenadas o troncocónicas con baquetones que decoran la pared externa bajo el borde, a veces rellenos aquéllos de filas de líneas incisas verticales. La industria lítica es rica, con cuchillos de sílex de filo retocado, algunos raspadores (?) y restos de lascado. Aunque no se han documentado útiles metálicos existen en el sitio evidencias de metalurgia de cobre a partir de glóbulos de ese metal fundido adheridos a la cara interna de fragmentos cerámicos, lo que parece evidencia indiscutible de su obtención por medios de fundición muy arcaicos. En cuanto a los restos alimenticios señalar la presencia de bóvidos, ovicápridos, cerdos y moluscos, si bien deben mantenerse ciertas reservas dado el carácter del hallazgo.

$\mathrm{El}$ abrigo de Son Matge, en las inmediaciones de la ciudad de Valldemossa, ya en el comienzo de las tierras altas de la isla, es otro de los yacimientos de referencia. Son Matge constituye un sitio único, por el momento, para el estudio de la prehistoria de Mallorca, con una larga secuencia estratigráfica que abarca desde los primeros indicios de poblamiento de la isla hasta la romanización efectiva del 
territorio. Su situación, dominando un estrecho paso que da acceso al valle de Valldemossa, en una zona donde suele haber agua de manera continua, parece muy propia para el asentamiento humano. Quizá ello explique su uso continuado como reflejo de la ocupación del territorio inmediato, si bien unas veces el yacimiento se utilizó como lugar de habitación y en otras épocas como necrópolis.

Los materiales de filiación campaniforme aparecen en este yacimiento con fechas comprendidas entre 1720 y 2030 a. C. (Fernández-Miranda y Waldren, 1979), con otras más intermedias. La mayor parte de los fragmentos cerámicos hallados se decoran con líneas en zig-zag, aunque también están presentes los motivos ajedrezados y de dientes de lobo. Con ellos han aparecido otros materiales, como punzones sencillos de bronce, un peine de marfil que reproduce los motivos decorativos de ajedrezado de la cerámica campaniforme y muñequeras de arquero. Pero quizá lo más destacable sea la documentación, sobre cerámicas de superficies decoradas con motivos campaniformes, de evidentes indicios de metalurgia de cobre en forma también de glóbulos de metal fundido adheridos a las caras internas (Waldren, 1979).

El conjunto de niveles de Son Matge con cerámicas y otros elementos de tipo campaniforme parecen corresponder a un momento en que el yacimiento es utilizado como lugar de habitación. Se corresponde con los niveles 22 a 17 de la sistematización de Waldren (1982, págs. 160 y 161), tras un nivel de transición, el 23, que separa este bloque del subyacente, caracterizado por la presencia de restos de Myotragus balearicus en un ambiente neolítico final exento de cerámicas con decoración campaniforme (debe señalarse que el nivel 24 del yacimiento - IX/X de las sistematizaciones más generales - fue al principio erroneamente considerado dentro de la fase campaniforme con su fecha 2070 a. C.). En conjunto se distinguen varios niveles de fuego, probables restos de hogares e indicios de desechos de alimentación a base de huesos de cerdo, cabra y oveja. Se trata, sin duda, de animales introducidos en la isla, pues no existen allí en estado salvaje, lo que provocó que el Myotragus dejara de ser cazado, tras los intentos de domesticarlo, actividades que en Son Matge quedan probadas a lo largo del cuarto y tercer milenios, a partir de 2700 a. C. en relación con cerámicas de filiación neolítica final (FernándezMiranda y Waldren, 1979, págs. 354 y 355). Parece evidente que la irrupción de los productos de tipo campaniforme en Son Matge debe ponerse en relación, al menos cronológica, con el comienzo de la metalurgia en la isla y la introducción de distintas especies de animales domésticos que van a constituir la base ganadera fundamental en la estrategia alimentaria de la población pretalayótica de Mallorca.

El último poblado mallorquín del que se tiene buena información para esta época es el de Son Ferrandell-Oleza, cerca también de Valldemossa y no muy alejado del abrigo de Son Matge (Waldren, 1983 y 1986, págs. 92 y ss.). Son Ferrandell-Oleza en un vasto complejo arqueológico, todavía en curso de excavación y estudio, que ha proporcionado restos de poblamiento desde época pretalayótica hasta el período talayótico final, aunque quizá no de manera tan continuada y estratigráficamente seriada como aparecen en Son Matge. Ocupa la ladera de un valle en un terreno 
que también es apto para la agricultura pero, sobre todo, para la ganadería. La ocupación más antigua está formada por una construcción rectangular amurallada de aproximadamente $60 \times 45 \mathrm{~m}$. En su interior se localizan dos cabañas alargadas de forma rectangular y otra de cabecera absidal, que recuerda los tipos constructivos de las navetas de habitación, así como un canal cuidadosamente ejecutado en suave desnivel para suministrar agua al poblado. La muralla posee torres en dos de sus esquinas. Esta construcción debió tener una vida relativamente larga, a lo largo de toda la fase pretalayótica, y sin duda sus murallas, a juzgar por las dataciones absolutas que las fechan, fueron rehechas en distintas ocasiones. La más antigua, que se sitúa al este, fue erigida en torno a 2000 a. C. (BM-1843: $3950 \pm 60$, en Waldren, 1986, tabla 16 c), según un resultado C-14 obtenido en la base del muro, mientras que los depósitos debidos a la ocupación humana que ciegan el canal, en particular huesos de animales y restos cerámicos, tienen fechas hacia 1200-1100 a. C., lo que significa unos ochocientos años de ocupación del sitio. Desde el punto de vista que nos ocupa, interesa la excavación en el sector oeste del recinto, donde han aparecido, en un relleno de materiales pretalayóticos, varios fragmentos de cerámica campaniforme junto con industria lítica de cuchillos en sílex con retoque lateral (Waldren, 1983, fig. 2). La datación de este conjunto arqueológico mediante C-14 es 1770 a. C. para el estrato inferior y 1750 para el siguiente (BM-1981: $3720 \pm 35$ y QL-1592: 3700 B.P. \pm 30, respectivamente). En Son Ferrandell-Oleza existen, asimismo, indicios de metalurgia desde el momento más antiguo de la vida del poblado, constatándose también en fases posteriores la obtención del cobre, con cuya manufactura probablemente hay que relacionar el sistema hidráulico del sitio.

Los otros dos yacimientos con cerámica campaniforme claramente definida poco ayudan a incrementar los datos hasta aquí obtenidos. Uno es la Cova dels Bous, un hallazgo casual al que ya he hecho referencia en la introducción a estas líneas. El otro es Son Torrella, una cueva natural en la zona más alta de la isla, en cuyo interior Enseñat recuperó una serie de materiales hacia el año sesenta (Enseñat, 1961). Este yacimiento debía ser muy rico y de gran interés pero los deficientes trabajos en él realizados facilitan poco su interpretación. Entre la cerámica encontrada había fragmentos de clara filiación campaniforme decorados con bandas de líneas entrecruzadas, bandas en zig-zag y dientes de lobo; junto a ellas otras de líneas más sencillas parecen señalar la perduración en la ocupación del sitio, sino la coexistencia de estilos decorativos diversos. Se recogieron asimismo botones de perforación en $\mathrm{V}$, cuchillos de sílex con el borde retocado y un buen número de lascas. La mayor parte de la fauna estaba compuesta, al parecer, por ovicápridos, lo que se comprende perfectamente dado el ambiente de alta montaña en que se encuentra el yacimiento. Son Torrella parece ser un lugar de habitación esporádica, quizá un refugio de pastor apenas acondicionado y bastante incómodo, pues presenta numerosas escorrentías de agua y su suelo es quebrado sin que se perciba obra alguna de acondicionamiento. Hay sin embargo autores que lo han interpretado de distintas maneras, como santuario o, en algún momento, necrópolis (véase, a estos efectos, Cantarellas, 1972 a, pág. 43). 


\section{Isla de Menorca}

Hasta ahora no ha sido localizado en Menorca ningún yacimiento donde con claridad se puedan clasificar conjuntos materiales de tradición o filiación campaniforme. De manera aislada se puede citar el pequeño puñal (?) procedente del sepulcro megalítico de Ses Roques Llisses, cerca del poblado de Torre d'en Gaumés, en Alaior (Rosselló Bordoy, Plantalamor Massanet y López Pons, 1980). Apareció en el sector absidal de la cámara, mientras que en otros puntos se recogieron un botón de perforación en $\mathrm{V}$, un posible brazalete de arquero fragmentado y pedazos de vasos cerámicos globulares, concoides o troncocónicos, con algunas carenas.

El puñal en cuestión mide $6,5 \mathrm{~cm}$. de longitud y está terminado por percusión mediante martilleo. Una pieza próxima, tanto por su tipología como por su tamaño, fue hallada hace años en Es Rafal d'es Toro, de donde pasó a la colección particular de Martínez Santa-Olalla, desconociéndose su paradero actual (Martínez SantaOlalla, 1935, págs. 49 y 50). Estos dos puñalitos parecen corresponderse con los de lengüeta de tipo campaniforme, una filiación que, por el contexto en que aparece, no parece desdeñable para el caso del ejemplar hallado en la tumba megalítica de Ses Roques Llisses.

\section{Islas de Ibiza y Formentera}

Un verdadero puñalito de lengüeta triangular se conserva en el Museo de Ibiza desde 1907 y procede, al parecer, de la isla, sin que se conserve alguna otra noticia sobre su descubrimiento (Fernández, 1974, pág. 64). Mide $117 \mathrm{~mm}$. de longitud, 40 de anchura y su espesor máximo es de $2,5 \mathrm{~mm}$. A lo largo de todo el borde de su hoja presenta nítidas huellas de martilleo, lo que indica la existencia de unos claros rebajes laterales intencionados que provocaron, hacia el extremo distal, la aparición de un nervio central que no existe en el resto de la hoja. Presenta lengüetas conseguidas por estrangulamiento, aparentemente en frío, y bajo ellas, en el nacimiento de la lámina, huellas del arco de enmangue.

Además de ese claro hallazgo de filiación campaniforme debe recordarse que la aparición en el sepulcro megalítico de $\mathrm{Ca} \mathrm{Na}$ Costa, en Formentera, de un par de pequeños trozos cerámicos de estilo campaniforme (Fernández, Plantalamor, Topp, 1976, pág. 125), uno con decoración de banda rellena de líneas entrecruzadas y otro con dientes de lobo. Aparecieron asociados a cuencos y vasijas troncocónicas, botones de perforación en $\mathrm{V}$, restos de un brazalete de arquero y algunas lascas de sílex. La fecha obtenida por C-14 a partir de uno de los enterramientos hallados fue 1320 B.C. (BM-1677: $3270 \pm 80$ ). El sepulcro de $\mathrm{Ca} \mathrm{Na}$ Costa es un monumento ciertamente singular en el contexto de la prehistoria balear, separándose de otros megalitos conocidos en las islas por su concepción y calidad constructiva. Está formado por una cámara central poligonal de siete lajas más otra perforada que sirve de relación con el pasillo; por fuera presenta tres círculos bien trazados, el intermedio con lajas hincadas dispuestas en sentido radial y todos con un murete 
de contención en la línea externa. La cámara funeraria contenía un mínimo de seis individuos inhumados, según sus excavadores, en momentos distintos pero aparentemente dentro de un lapso corto de tiempo.

\section{Discusión e interpretación de estos hallazgos}

No son ciertamente muchos ni muy variados los objetos que en las Islas Baleares pueden ser claramente relacionados con las producciones consideradas característicamente campaniformes. Parece que el archipiélago, dentro de los contactos que la aparición de tales piezas significa, debió ocupar, probablemente por su propio carácter geográfico, una posición poco significativa en calidades y cantidades, aunque quizá sí relevante desde el punto de vista de la evolución histórica de las islas, en particular - por obvias razones de información- en el caso de Mallorca. Tal situación sin duda ha sido una de las causas que ha llevado a los autores a ocuparse, sobre todo, dé cuestiones tipológicas, y, dentro de ellas, a las relacionadas con las producciones cerámicas, quedando prácticamente inéditos el resto de los objetos que las acompañan y los modelos de ocupación del territorio que se corresponden sincrónicamente.

La cerámica campaniforme en Mallorca, tradicionalmente denominada incisa por la precaución a considerarla como tal debido a la ausencia de otros elementos clave de ese complejo tecnológico e incluso de la variedad formal que le es característica, por ejemplo, en la Península Ibérica, fue sistematizada por primera vez por Rosselló Bordoy ya citado. Estableció un grupo de mejor calidad técnica en la factura y motivos decorativos de relativa complejidad, frente a otro más deficiente en el que predominan los motivos ornamentales sencillos. Años después Veny (1968, págs. 407 y ss.) mantienen los dos tipos, si bien introduce notables variantes en los criterios clasificatorios. En síntesis, el grupo A de Veny se caracteriza por una decoración fina y apretada, con motivos no muy complicados pero repetidos y llenos, aplicados preferentemente sobre vasos de forma concoidea o semiesférica, mientras que el grupo B se define por la decoración a base de trazos finos y más burdos aplicados preferentemente sobre vasos troncocónicos. El grupo A correspondería a una etapa campaniforme, mientras que el B es postcampaniforme. La última clasificación es obra de Cantarellas, también citada en la introducción, quien distingue dos grandes grupos con valores cronológicos, Incisa I (2000-1800 a. C.) e Incisa II (1900-1450 a. C.) y dentro de este segundo tres variantes según estilos decorativos y, sobre todo, formas cerámicas que los contienen. En los yacimientos de la fase Incisa I el metal está prácticamente ausente y, en contrapartida, se desarrolla una importante industria en sílex, que queda reducida prácticamente a series de lascas atípicas en la fase Incisa II, donde ya sí aparece el metal.

A mi modo de ver las cosas el temor a aceptar la existencia de materiales de filiación campaniforme, que yo mismo desarrollé (Fernández-Miranda, 1978) y que tan sólo Veny, aunque tímidamente, defendió (Veny, 1968, pág. 411), complicó en sobremanera las cosas, creando un falso grupo, el de las cerámicas incisas, que, 
indiscutiblemente, resultaba operativo en la medida en que de esa manera se evitaba formalizar una "fase" campaniforme en la secuencia histórica de la isla, con los problemas que tal operación comporta, aunque a la postre la cuestión tampoco encontró solución por ese camino. En origen el error residía en hablar de las cerámicas incisas como un grupo con entidad cultural, aunque su extensión y características variaran según autores. De acuerdo con los datos que hoy en día se poseen no parece descabellado mantener la filiación campaniforme exclusivamente para los yacimientos que aparecen en este trabajo, que en el caso de Mallorca se corresponden con un momento antiguo dentro del período pretalayótico, fechable, en términos absolutos, entre el 2030 a. C. de Son Matge y el 1750 a. C. de Son FerrandellOleza, es decir con una duración aproximada de unos trescientos años. Yacimientos con tipos cerámicos decorados a base de líneas incisas verticales, como en $\mathrm{Sa}$ Canova, sobre vasos de forma troncocónica, u otros como Es Corral d'es Porc con su vaso bitroncocónico, o son Bauçá, con un vaso globular que se adorna a base de improntas de caña circulares (Cantarellas, 1972 a), no deben ser puestos en conexión con las producciones estrictamente campaniformes, aunque en casos puedan ser sincrónicas y aunque todo parezca indicar que se trata de piezas ligeramente más tardías.

En cuanto a las posibles conexiones geográficas de estas cerámicas mallorquinas la cuestión parece compleja pues, a primera vista, la sensación que ofrecen es la de mezclar estilos decorativos y formas que en las áreas más inmediatas, la Península Ibérica y el sur de Francia, se encuadran en grupos diferenciales. Los tipos cerámicos dominantes son los cuencos y escudillas, faltando hasta ahora en el archipiélago los modelos cerámicos más complejos, como los recipientes altos de cuello estrechado o las cazuelas tipo Ciempozuelos. Ciertamente ello puede deberse al bajo número de piezas hasta ahora encontradas que permiten su restitución. Algunos hallazgos recientes, por ejemplo, en Son Ferrandell-Oleza parece que pueden responder a formas carenadas. Ciertos fragmentos parecen corresponder al estilo decorativo marítimo y hay platos con ónfalo que recuerdan producciones tipo Carmona, pero la mayoría de los trozos decorados apuntan sus paralelos hacia el denominado grupo Salamó o del campaniforme catalán, así como a las producciones del Pirineo francés (véase un cuadro de similitudes decorativas en FernándezMiranda y Waldren, 1979, fig. 3). Una cosa parece, en consecuencia, clara y es que estas producciones cerámicas campaniformes de Mallorca parecen tener una indiscutible personalidad, que aumenta si se valoran pastas y fabricaciones, de indiscutible producción local. Quizá todo ello fuera suficiente para hablar de un específico grupo campaniforme balear, que se definiría por la casi exclusiva producción de cuencos y escudillas con decoraciones de bandas, ajedrezados, líneas en zig-zag y dientes de lobo y, excepcionalmente, motivos radiales estelares confluyendo hacia el fondo exterior del recipiente; en ciertos casos es posible que existan vasos troncocónicos, lo que, al fin y al cabo, también vendría a constituir una marcada característica local. Así el "grupo balear" que Castillo con un solo fragmento creó en los años veinte recobraría vida, algo mejor caracterizado tanto cualitativa como cuan- 
titativamente y, desde luego, cambiando con casi total seguridad la línea de relación que entonces se proponía.

En cuanto al resto de los objetos asociados a las cerámicas poco más permiten decir. Los útiles metálicos son muy sencillos y responden a tipos que están generalizados por amplias áreas geográficas, y lo mismo cabe decir de las llamadas muñequeras de arquero. Quizá sea más significativa la presencia de botones de perforación en $\mathrm{V}$ de forma piramidal, junto a otros más sencillos triangulares. Pueden poner de nuevo en relación al archipiélago, o, al menos, a Mallorca, con el área catalana, donde esa clase de botón es bien frecuente en ambientes campaniformes (Harrison, 1980, págs. 143 y 144). Esta asociación de productos cerámicos decorados con botones de perforación en $\mathrm{V}$, muñequeras de arquero y ciertos tipos cerámicos -e incluso el hecho mismo de la aparición de la metalurgia- lleva de nuevo al planteamiento de la relación que puede existir entre todos esos objetos a la hora de valorar conjuntamente los elementos culturales considerados genuinamente campaniformes. Pero esa es cuestión que excede de lo que aquí se trata ahora.

Un asunto sin duda relevante es recapitular sobre el tipo de yacimientos en que aparecen estos materiales de más clara filiación campaniforme. La mayor parte de los casos conocidos son lugares de habitación. En realidad si excluimos el puñalito de Ses Roques Llisses, en Menorca, y los dos pequeños fragmentos cerámicos del megalito de $\mathrm{Ca} \mathrm{Na}$ Costa, en Formentera, tan sólo las cistas del abrigo de Son $\mathrm{Ga}$ llard representan adecuadamente esa clase de materiales en un yacimiento de corte funerario. Por el contrario los lugares de habitación - todos en Mallorca - son de variada tipología. En dos casos, Es Velar y Son Ferrandell-Oleza, parece tratarse de poblados relativamente extensos; $\mathrm{Ca} \mathrm{Na}$ Cotxera, sin embargo, es un hábitat aislado al aire libre, mientras que Son Torrella y Son Matge indican la existencia de hábitat en cuevas y abrigos naturales. Y todo ello, según parece, sincrónicamente.

En la formulación de la secuencia de la prehistoria de Mallorca (FernándezMiranda, 1978) se admitía que los poblados, como ejemplo de asentamiento colectivo, constituían un fenómeno relativamente tardío, en un momento ya avanzado de la cultura talayótica. Cierto que algunas aglomeraciones de construcciones naviformes, como ocurre, por ejemplo, en Bóquer (Fernández-Miranda, 1978, fig. 21), parecían anunciar la existencia de concentraciones de cierta envergadura en época pretalayótica, pero ni parecen corresponder a un poblado organizado como tal ni existen evidencias de que en ese caso, o en otros posibles, todas las construcciones visibles hayan estado sincrónicamente en uso. Lo normal es que las navetas de habitación se presenten aisladas o en pequeños grupos, y ello tanto en Mallorca como en Menorca. A un lado el poblado de Es Velar, por las limitaciones que presenta para su interpretación, Son Ferrandell-Oleza parece romper ese esquema proporcionando la existencia de un poblado de buenas dimensiones en un momento antiguo de la fase pretalayótica y en un terreno, el de montaña, que no es evidentemente el más apto para desarrollar un tipo de agricultura que posibilite una cierta acumulación de personas. Por el contrario $\mathrm{Ca} \mathrm{Na}$ Cotxera, en un ambiente teóricamente mucho más favorable, es, al parecer, una vivienda aislada, al estilo de las navetas de habitación tipo C'an Alemany (Enseñat, 1971) o del uso de abrigos y 
cuevas por un grupo humano de dimensiones reducidas. Si Son Ferrandell-Oleza es ya un poblado en ese momento, lo que por el momento parece muy probable aunque no indiscutible hasta tanto no terminen los trabajos en el yacimiento, se hace necesario buscar las razones económicas que lo hicieron posible. Tal vez una explotación agraria del valle inmediato, en una zona que teóricamente es favorable para la recogida de aguas, 'según ya se comentó a propósito de la instalación hidráulica que posee el poblado, o quizá la existencia en sus inmediaciones de recursos mineros, con la consiguiente actividad metalúrgica demostrada en el yacimiento en las áreas de ocupación que han proporcionado materiales campaniformes o sincrónicos a ellos. $\mathrm{O}$ acaso haya que considerar ambas posibilidades al unísono, pues para las dos el agua constituye un elemento de primera necesidad.

En cuanto a los lugares de uso funerario poco se puede decir. Las cistas de Son Gallard constituyen, por el momento, un unicum en el archipiélago balear, donde los enterramientos sincrónicos conocidos parecen ser los de tipo dolménico. Hasta ahora están documentados en el ámbito de las Islas Baleares cuatro megalitos en Menorca, uno en Mallorca y otro en Formentera, es decir un total de seis al que habría que sumar quizá la extraña construcción de C'an Sargent, en Ibiza, muy deteriorada y que con materiales relativamente antiguos ha proporcionado una fecha C-14 de 800 B.C. difícil de encajar (Fernández, 1984, pág. 592), salvo que se acepte una larga perduración para esa clase de construcciones en el marco de las Pitiussas. En dos de ellos, como ya se ha dicho, han aparecido materiales de filiación campaniforme; aceptada la similitud de los restantes materiales con los que aparecen en los demás sepulcros megalíticos del archipiélago puede suponerse que todos ellos resultan ser sincrónicos con la época de referencia. De tal manera que las dos cistas de Son Gallard, el dolmen de Son Bauló de Dalt en Mallorca y los cuatro de Menorca, en realidad las cinco cistas evolucionadas (Plantalamor, 1976-77) constituirían los ejemplos del ritual funerario pretalayótico más antiguo y sincrónico con los objetos campaniformes de Mallorca y Menorca. El caso de $\mathrm{Ca} \mathrm{Na}$ Costa puede responder a una perduración del ritual con elementos que perviven paralelizables en Mallorca y Menorca, las cerámicas, y otros que innovan, el modelo constructivo. Y una cronología bastante más tardía, al menos por la fase de utilización que fecha el análisis C-14 comentado más atrás.

Otro asunto asimismo de interés es la relación que ese momento, y esos elementos culturales, pudieron tener con el comienzo de la metalurgia del cobre en las islas. Si de nuevo se dejan en entredicho los datos procedentes del poblado de Es Velar, la más antigua evidencia de metalurgia en las Baleares corresponde hoy por hoy a los yacimientos de Son Matge y de Son Ferrandell-Oleza, siempre en ambientes arqueológicos caracterizados por la presencia de cerámicas decoradas de tipo campaniforme. En Son Matge (Waldren, 1979) han aparecido en las caras internas de algunas de ellas glóbulos de cobre fundido, prueba de la utilización de recipientes cerámicos como contenedores de mineral en un primer proceso de reducción dentro de un horno primitivo de pozo abierto en la tierra. No debe extranar que la minería y la metalurgia más antiguas del cobre se vinculen a la zona de Valldemossa, pues por allí existen varios yacimientos de minerales aptos, como 
ocurre en otros puntos próximos a lo largo de la Sierra de Tramuntana. Cabría la hipótesis de que estos poblados de montaña facilitaran el mineral a los de la zona baja, o quizá ya las sencillas producciones tipo punzones de cobre. Sería la prueba del primer intercambio de productos en la isla de Mallorca y explicaría, al menos en parte, la relativa concentración de población que el registro arqueológico viene detectando para los primeros tiempos de la fase pretalayótica en la zona alta de la isla.

Distinto problema plantea el puñal de lengüeta de Ibiza, puesto que en esa isla no existen minerales de cobre. La pieza es un cobre arsenical (Cu: 97,14 \%; As: $1,76 \%$ ) que o bien fue importada o bien hasta Ibiza se exportó el mineral necesario para su fabricación. El puñalito triangular de C'an Sargent, un bronce con 89,07 de $\mathrm{Cu}$ y $9,93 \%$. de Sn, confirma en fechas posteriores, aunque se trate de una pieza muy arcaica, pero aún anteriores, en todo caso, a la llegada de los púnicos a la isla, la existencia de relaciones exteriores, en este caso de nuevo o la pieza manufacturada o los dos minerales básicos que se usaron para su fabricación.

Con esos dos ejemplares debe plantearse la cuestión del origen de la metalurgia en las islas. Complementariamente señalar que en la isla de Menorca, donde si hay minerales de cobre que permitirían fabricar los puñales de Ses Roques Llisses y Es Rafal d'es Toro, no existen minerales de plomo, y sin embargo los indicios más antiguos de prácticas metalúrgicas en la isla, documentadas en la naveta de habitación de Son Mercer de Baix, demuestran la existencia de ese componente tanto en una de las piezas manufacturadas, un puñal, como en un lingote dispuesto para ser fundido. De manera que si bien en Mallorca el comienzo de la metalurgia pudo ser una invención local tampoco podemos descartar que el mismo fenómeno que provocó la irrupción de elementos campaniformes en la isla llevara consigo esa tecnología. Sería la vuelta al problema de las relaciones entre cerámicas campaniformes, botones de perforación en $\mathrm{V}$, muñequeras de arquero y otros utensilios al uso con la expansión de la metalurgia calcolítica por extensas zonas del Mediterráneo occidental, una cuestión ésta que aún se encuentra sometida a debate. En los casos de Menorca e Ibiza, aunque el primero en fechas posteriores, parece probado que fueron necesarias determinadas relaciones exteriores para lograr la obtención o el disfrute de determinados objetos. Por lo que se refiere estrictamente al asunto de las cerámicas campaniformes basta contemplar el mapa de los hallazgos a lo largo de toda la cuenca del Mediterráneo occidental e islas en ella situadas para comprender que a comienzos del segundo milenio a. C. los contactos por aquella debían ser si no frecuentes sí algo tampoco excesivamente accidental. Esas relaciones debieron, entre otras cosas, provocar la aparición de cerámicas y otros útiles de tipo campaniforme en las Islas Baleares, muy probablemente desde las costas de Cataluña y el Languedoc.

Cales Coves (Menorca), septiembre 1986

Agradecimiento. El autor desea agradecer públicamente a William $\mathrm{H}$. Waldren los datos que sobre los asuntos que aquí se tratan le facilitó a lo largo de las distintas ocasiones en que ha sido siempre cariñosamente acogido en el Deià Archaeological Museum and Research Center (DAMARC) en Deià, isla de Mallorca. 


\section{BIBLIOGRAFIA}

Cantarellas Camps, C. (1972 a): Cetámica incisa en Mallorca, Palma.

Cantarellas Camps, C. (1972 b): Excavaciones en Ca Na Cotxera (Muro, Mallorca). Noticiario Arqueológico Hispánico, Prehistoria I, págs. 177 a 226.

CARreras, J. y CovaS, J. (1984): La ceràmica incisa a Santanyí. Boletín de la Sociedad Arqueológica Luliana, XL, págs. 3 a 38.

CASTILlO, A. (1928): La cultura del vaso campaniforme, Barcelona.

ENSEÑAT, B. (1961): Noticia sobre el hallazgo de unas cerámicas arcaicas del estilo del vaso campaniforme. VII Congreso Nacional de Arqueología.

ENSEÑAT, C. (1971): Excavaciones en el naviforme Alemany (Magaluf). Noticiario Arqueológico Hispánico, XV, págs. 37 a 73 .

FERNÁNDEZ, J. (1974): Hachas de bronce halladas en Ibiza y Formentera. Prehistoria y Arqueología de las Islas Baleares. VI Symposium de Prehistoria peninsular, págs. 63 a 71.

FERnÁNDEZ, J. (1984): The Prehistory of Eivissa and Formentera. Biogeography and Ecology of the Pityusic Islands, La Haya, págs. 565 a 595.

Fernández, J.; Plantalamor, L. y TOPP, C. (1976): Excavaciones en el sepulcro megalítico de Ca Na Costa (Formentera), Mayurga, 15, págs. 109 a 138.

FernándeZ-Miranda, M. (1978): Secuencia cultural de la Prehistoria de Mallorca, Madrid.

FERNÁNDEZ-MIRANDA, M. y GARRALDA, M. D. (1978): Contribución al estudio de las poblaciones prehistóricas de la isla de Mallorca. I Symposium de Antropología biológica de España, págs. 87 a 104.

FERNÁNDEZ-MiRANDA, M. y WALDREN, W. H. (1979): Periodificación cultural y cronología absoluta en la prehistoria de Mallorca. Trabajos de Prehistoria, 36, págs. 349 a 377.

HARRISON, R. J.: The Beaker Folk, Londres.

Martínez Santa-Olalla, J. (1935): Elementos para un estudio de la cultura de los talayots en Menorca. Actas y Memorias de la Sociedad Española de Antropología, Etnografía y Prehistoria, XIV, págs. 5 a 66 , XIX láms.

PLANTALAMOR, L. (1976-77): Algunas consideraciones sobre los sepulcros megalíticos de Menorca. Sautuola, II, págs. 157 a 173.

Rosselló Bordoy, G. (1960): Cerámicas incisas de Mallorca. Studi Sardi, XVI.

Rosselló Bordoy, G.; Plantalamor Massanet, L. y López Pons, L. (1980): Excavaciones arqueológicas en Torre d'en Gaumés (Alayor, Menorca). I. La sepultura megalítica de Ses Roques Llises. Noticiario Arqueológico Hispánico, 8, págs. 71 a 126.

VENY, C. (1968): Las cuevas sepulcrales del Bronce antiguo de Mallorca, Madrid.

WALDREN, W. H. (1979): A Beaker workshop area in the Rock Shelter of Son Matge, Mallorca. World Archaeology, 11, 1, págs. 43 a 67.

WALDREN, W. H. (1982): Balearic prehistoric Ecology and Culture, Oxford.

WALDREN, W. H. (1983): A Beaker Settlement from the Balearic Island of Mallorca, Spain. The Settlement Complex of Ferrandell-Oleza. Valdemosa, Mallorca, Spain. Homenaje al Profesor Martín Almagro Basch, tomo II, págs. 177 a 183.

Waldren, W. H. (1986): The Balearic Pentapartite Division of Prehistory, Oxford. 